\title{
Regional estimation and validation of remotely sensed evapotranspiration in China
}

\author{
Chesheng Zhan ${ }^{\mathrm{a}}$, Jian Yin ${ }^{\mathrm{a}, \mathrm{b}, *}$, Feiyu Wang ${ }^{\mathrm{a}, \mathrm{d}, * *}$, Qingqing Dong ${ }^{\mathrm{c}}$ \\ ${ }^{a}$ Key Laboratory of Water Cycle E Related Land Surface Processes, Institute of Geographical Sciences and Natural Research, CAS, Beijing 100101, China \\ b School of Resources and Environment, Anqing Normal University, Anqing 246011, China \\ c College of Water Sciences, Beijing Normal University, Beijing 100875, China \\ d University of Chinese Academy of Sciences, Beijing 100049, China
}

\section{A R T I C L E I N F O}

\section{Article history:}

Received 16 July 2014

Received in revised form 13 April 2015

Accepted 27 April 2015

Available online 16 May 2015

\section{Keywords:}

Evapotranspiration

Remote sensing

SEBS-China

Simulation

China

\begin{abstract}
A B S T R A C T
Accurate estimates of evapotranspiration (ET) are important to skillful predictions of runoff, soil moisture and crop productivity. In this study, ET was simulated over three years in China by integrating remotely-sensed data with the simulation model SEBS-China. SEBS-China was developed from the Surface Energy Balance System (SEBS) model that is used to estimate atmospheric turbulent heat fluxes and evaporative fraction through satellite-derived radiation fluxes and land surface temperatures coupled with near-surface meteorological variables. Simulated regional ET was first evaluated using experimental data from the Changbai Mountains and five river basins in China. Average annual simulated ET agreed well with the observed data, based on the detailed results through comparative validation. Regional ET derived from SEBS-China was then compared with the simulated ET from the Common Land Model (CoLM). Both of the models simulated regional ET well, with a few overestimated areas such as in the Qinghai-Tibet Plateau, YanShan and South Daxing'anling Mountains. We conclude that more efficient ET estimation can be achieved through combining remotely-sensed information with land surface models.
\end{abstract}

(c) 2015 Elsevier B.V. All rights reserved.

\section{Introduction}

Evapotranspiration (ET) consumes over half of the solar radiation absorbed by land surface to evaporate, and transfers $70 \%$ of precipitation to water vapor across the world (Jung et al., 2010; McMahon et al., 2013). Detailed knowledge of ET is vital for monitoring regional and global climate change through the hydrological cycle, and its estimation has significant applications in agriculture especially in areas such as runoff prediction, soil moisture prediction, crop yield prediction, and land use planning (Bastiaanssen et al., 2007; Kustas and Norman, 1996). The conventional method that uses point measurements to estimate ET values is only representative of local areas and cannot be extended to large areas due to the dynamic nature and regional variation of ET (Courault et al., 2003). Remote sensing has proven to be the a suitable approach for large-area estimates of ET because satellite remote sensing is an efficient technology that can provide representative

\footnotetext{
* Correspondence to: J. Yin, Key Laboratory of Water Cycle \& Related Land Surface Processes, Institute of Geographical Sciences and Natural Research, CAS, Beijing 100101, China.

** Correspondence to: F. Wang, University of Chinese Academy of Sciences, Beijing 100049, China.

E-mail address: yinjianbnu@163.com (J. Yin).
}

parameters such as radiometric surface temperature, albedo and vegetation index in a globally consistent and economically feasible manner (Choudhury and Fung, 1989; Kustas et al., 2003).

Remote sensing data with the increasing imagery resolution is a useful tool to provide much more information at various scales. A variety of methods have been developed to use that information in land surface flux estimation schemes. Physically based analytical approaches derived from the Penman-Monteith resistance model and surface energy balance equations are the most common methods. They either use radiometric surface temperature to calculate the sensible heat flux $\mathrm{H}$ and then use the energy balance equation to obtain ET value, or use radiometric surface temperature to calculate the crop water stress index to get the ET value (Bastiaanssen, 1995; Su and Menenti, 1999). However, the use of radiometric surface temperature as a substitute for the aerodynamic temperature in the original Penman-Monteith model leads to substantial error, especially over partial vegetation cover, because the radiometric surface temperature is a composite temperature consisting of soil and vegetation temperatures. Yet such error can be reduced in several ways: through an introduction of an extra-resistance (Kustas and Norman, 1996; Kustas et al., 1989), by switching from singleresistance models to a two-source model, by using more complicated multilayer models (Choudhury and Monteith, 1988; Lhomme et al., 1994a,b; Norman et al., 1995), by developing an empirical formula to 
estimate the aerodynamic temperature (Huang et al., 1993) and by incorporating a vegetation index within the model (Moran et al., 1994, 1996).

A Surface Energy Balance System (SEBS) for the estimation of atmospheric turbulent fluxes and surface evaporation using satellite earth observation data in the visible, near infra-red and thermal infrared frequency range has been designed for composite terrain with heterogeneous surfaces at a larger scale (Su, 2002). SEBS, applied to many case studies in Europe and Asia (Gokmen et al., 2012; Ma et al., 2014; Wang and Dickinson, 2012), has been shown as one of the most logical and accurate methods in ET estimation (Li et al., 2009). The accuracy of SEBS depends on the land surface physical parameters which is suitable to the study areas (Wood et al., 2003). In view of spatial and temporal scales and the complexity of underlying surface of China, SEBS needs to be revised and developed in the simulation of daily net radiation and some land surface physical parameters such as land surface temperature, the momentum roughness height and Leaf Area Index (LAI), to be more suitable for simulating ET of China's composite terrain.

This study aims to estimate the land surface ET in 1991, 1995 and 1999 in China based on satellite data, meteorological data, soil data and land cover data, by revising and developing the parameterization part of SEBS. The accuracy of the estimated results is tested by two aspects. The first is the comparison with the observed values in a representative experimental area (the Changbai Mountains) and five river basins (the Yellow River, the Yangtze River, the Haihe River, the Liaohe River and the Pearl River). The second is the cross-validation between the simulated regionally average ETs from SEBS-China and CoLM (Common Land Model).

\section{Methodology}

\subsection{The SEBS model}

The SEBS model was developed by the SEO-WaterCliEnt (Satellite Earth Observation Research into Water, Climate and Environment) group at the University of Wageningen Research Centre. Details of the model are provided in Su (2002) and Su and Jacobs (2001). The model consists of the following main components: 1) a set of land surface physical parameters, such as albedo, emissivity, temperature, and vegetation coverage, calculated from spectral reflectance and radiance data (Su et al., 1999); 2) a calculation of the roughness length for the heat transfer (Su, 2000); 3) the BAS (Bulk Atmospheric Similarity) theory (Brutsaert, 1999) calculating the friction velocity, the sensible heat flux and the Obukhov stability length; and 4) the SEBI (Surface Energy Balance Index) concept (Menenti and Choudnury, 1993) determining the evaporative fraction. The SEBS model requires three sets of input data. The first set includes the meteorological field data consisting of air temperature, air pressure, wind speed and humidity at the time of satellite transit. The second set is the daily average meteorological data including daily temperature, daily humidity and sunshine duration. The third set of data includes the land use and land cover (LUCC) classifications. The model processes of the SEBS are described as the following steps from (1) to (6), and the flowchart of the model and input data is shown in Fig. 1.

(1) Derive land surface parameters from remote sensing data given the corresponding atmospheric correction and geometric correction such as albedo, emissivity, surface temperature, fractional vegetation coverage, and vegetation index, and determine vegetation parameters according to land use and land cover data of the different seasons.

(2) Calculate the surface net radiation based on the surface energy balance, net long-wave radiation and short-wave radiation as an output of a large scale meteorological model or observation data of weather stations.

(3) Simulate the friction velocity, sensible heat flux and Obukhov stability length by the bulk parameterization approach. The criterion proposed by Brutsaert (1999) is used to determine whether MOS (Monin-Obukhov Similarity) or BAS (Bulk Atmospheric Boundary Layer (ABL) Similarity) stability correction functions

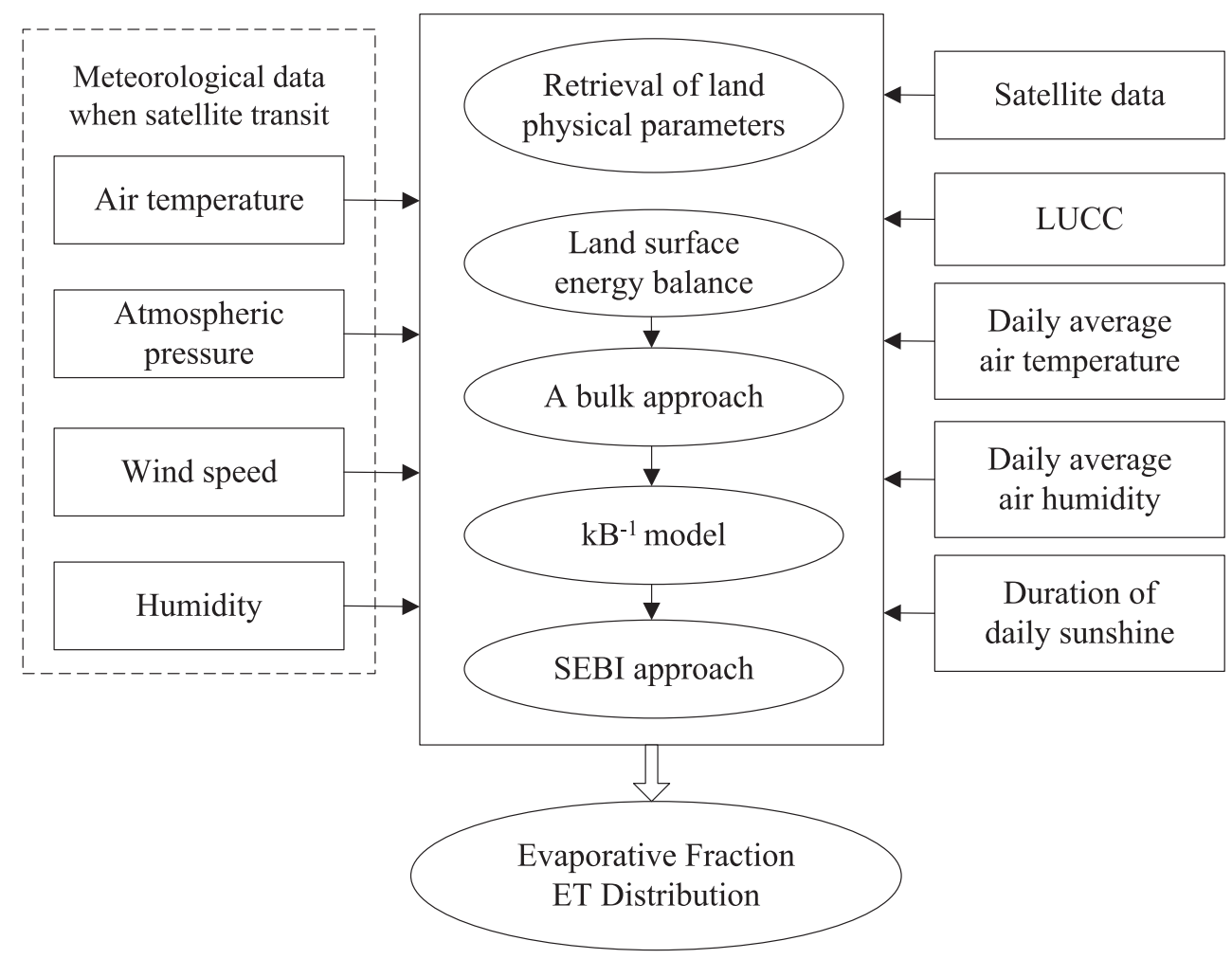

Fig. 1. Flowchart of SEBS model and input data. 
for momentum and sensible heat transfer are appropriate for a given situation.

(4) Establish spatially distributed $\mathrm{kB}^{-1}$ model to estimate the roughness length for heat transfer (Su, 2000).

(5) Simulate evaporative fraction based on the concept of SEBI and define the dry and wet limit conditions.

(6) Simulate daily actual regional ET by the evaporative fraction and the temporal scale transformation.

\subsection{Improvement of SEBS model}

According to China's specific regional characteristics, improvements have been made to the original SEBS model. A new submodule has been developed for daily net radiation simulation, and the retrieval of some land surface physical parameters has been improved (Zhan et al., 2007). The improvements include: 1 ) setting up a look-up table for momentum roughness length and zero plane displacement, 2) constructing a new air temperature parameterization with land surface temperature, 3) designing a new input interface of meteorological field at reference height and 4) adding a new module for total amount of daily net radiation.

The momentum roughness model adopted by the SEBS model is adapted to uniform dense grassland. In order to minimize the uncertainty caused by the retrieval of various vegetation parameters, such as fractional vegetation coverage and vegetation index, based on the component of SEBS, as a reference we used the field experiment database (http://www.ral.ucar.edu/research/land/technology/lsm/ noahlsm-v3.2/VEGPARM.TBL), which has been widely used around the world (Acharya et al., 2011; Ching, 2013; Ershadi et al., 2014; Gottschalck et al., 2005).

The land cover classification system of China was incorporated into the SEBS model, and monthly vegetation data for the different land covers were added, which enables the SEBS model to directly use parameters such as the roughness, zero-plane displacement, and vegetation height from the vegetation parameter database to reduce the simulation errors of the original model.

Research on the relationships between the measured air temperature, surface temperature, and vegetation fractional coverage for various types of land cover shows that the air temperature at the reference height $(2 \mathrm{~m})$ is closely related to some elements such as surface temperature, vegetation fractional coverage, and average apparent thermal inertia at the upper land surface (Zhan et al., 2007). Based on SEBS-China model, a simple air temperature-land surface temperature model suitable for China was established (Zhan et al., 2007):

$T_{\mathrm{a}}=28.5+0.1 T_{\mathrm{s}}-\left(f_{\mathrm{c}}+a\right) \xi$

where $f_{c}$ is the vegetation coverage; $a$ is a constant varying with $T_{s}$; and $\xi$ is the average thermal inertia for different land cover types and reflects the magnitude of thermal inertia of land surface.

\subsection{Data preparation and processing}

The input data mainly consists of the satellite data and ground observational data such as meteorological, hydrological, land cover and soil data. These data have a uniform coordinate system and a uniform temporal-spatial resolution. The daily meteorological data, including the maximum temperature (Tmax), the minimum temperature (Tmin), relative humidity $(\mathrm{RH})$, air pressure (Ps), wind speed at $10 \mathrm{~m}$ (U10), wind direction, daily precipitation (Pre) and sunshine hours (hsun), were provided by 400 weather stations evenly distributed throughout the whole country, whereas the remote sensing data consist of 10-day 8-km AVHRR composition data for 1991, 1995 and 1999 with five channels, NDVI, solar zenith angle, DOY (Day of Year), as well as corresponding auxiliary data, such as longitude, latitude and elevation.
The AVHRR data were extracted from the NOAA's Satellite Active Archive (SAA) according to a specified temporal-spatial range. Another relevant auxiliary set of data includes land cover and land use change (LUCC) in China in 1991, 1995 and 1999, which are provided by Data Centre for Resources and Environmental Sciences, Chinese Academy of Sciences (RESDC) (http://www.resdc.cn), and with a good data quality assessment to satisfy the spatial scale of this study (Liu et al., 2003, 2014). The LUCC data adopt a 3-level classification system. The first level includes six classes: farmland, forestland, grassland, water body, urban built-up land and barren land. The second level includes 25 classes based on natural attributes of land resources, while the third level includes eight classes based on geomorphological characteristics, type of land resources management, and the surface coverage.

The AVHRR products have been processed by image merging, radiation and atmospheric correction, and georeferencing. Moreover, the original GOODE projection has been transformed into the Albers conical equal area projection that is more suitable for China. The Inverse Square Distance (ISD) method was used to spatially interpolate meteorological data over China which includes daily average temperature, humidity, air pressure, and wind speed. Under the ArcGIS10.2 and the PCI/EASI calculation environment, the Lagrange interpolation method was adopted to carry out the temporal interpolation for each meteorological parameter. Original 1-km LUCC grid data was reclassified and resampled into the 8$\mathrm{km}$ LUCC grid data. All these processes resulted in a consistent projection system and temporal-spatial resolution for all input data.

\section{Results and validation}

\subsection{Modeling results of SEBS-China}

Primary outputs of the SEBS-China model are daily evaporative fraction, latent heat flux, sensible heat flux, net radiation flux and land surface physical parameters such as fractional vegetation coverage, land surface temperature, emissivity and albedo. These outputs were also synthesized into the 10-day ET, monthly ET and annual ET. Fig. 2 shows ET distribution of China in 1991, 1995, 1999, simulated by the SEBS-China model. The white areas represent deserts and water bodies. The purple areas that are mainly located on non-vegetated or sparsely vegetated region to the east of the Taklamakan Desert in the Xinjiang Autonomous Region, represent the low ET regions with ET value less than $200 \mathrm{~mm} \cdot \mathrm{a}^{-1}$. The red areas are mainly grasslands and parts of shrub distributed in northeastern and northern China, with relatively low ET values between $200 \mathrm{~mm} \cdot \mathrm{a}^{-1}$ and $400 \mathrm{~mm} \cdot \mathrm{a}^{-1}$. The brown and yellow areas representing shrubs, cropland and agriculture-forest mixed land are mainly located in China's northern forestland with medium ET values between $400 \mathrm{~mm} \cdot \mathrm{a}^{-1}$ and $600 \mathrm{~mm} \cdot \mathrm{a}^{-1}$. The dark gray, blue, and green areas representing cropland, parts of shrub and agriculture-forest mixed land are mainly located in the southern areas of the Qinghai-Tibet Plateau and the forestland of southern China, with high ET values more than $600 \mathrm{~mm} \cdot \mathrm{a}^{-1}$. The regionally average ET values of China were $593 \mathrm{~mm}$ in 1991, $650 \mathrm{~mm}$ in 1995 and $548 \mathrm{~mm}$ in 1999. The local maximum value appeared in the southern part of Yunnan Province, the southeast part of the Qinghai-Tibet Plateau and the central part of Hainan Province. In general, ET values in the south are higher than those in the north, and also are higher in the east than those in the west of China.

The simulated ET results show that the Yangtze and Pearl rivers locate in high ET regions, the Liaohe River is mainly located in the medium ET regions, the Yellow and Haihe rivers cross the medium and low ET regions, but their simulated ET in some places is significantly higher.

\subsection{Validation}

The Bowen Ratio Energy Balance (BREB), Eddy Covariance (EC) and Large Aperture Scintillometer (LAS) methods are experimental techniques widely used to measure surface flux of latent heat. Based on 

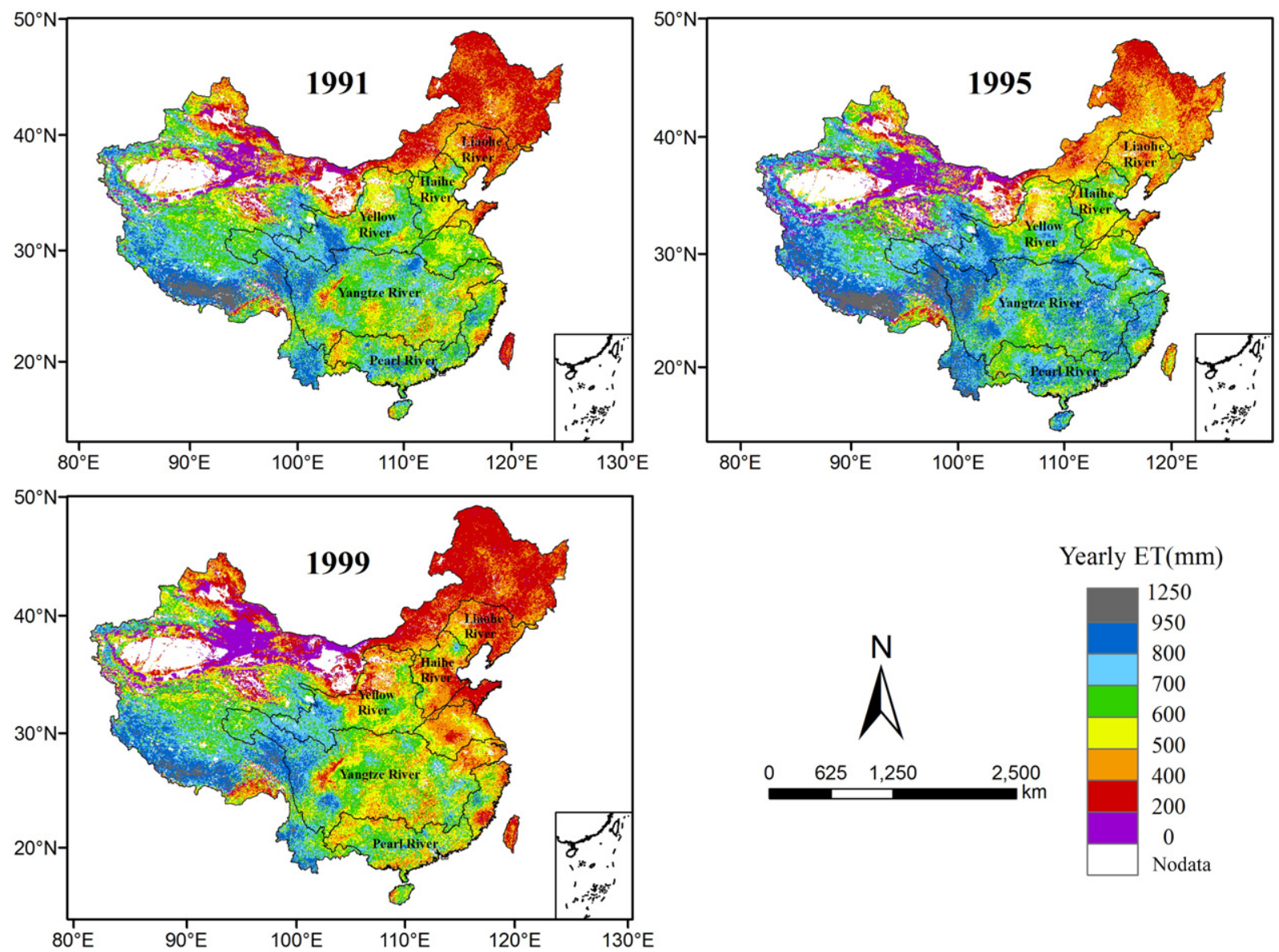

Yearly ET(mm)

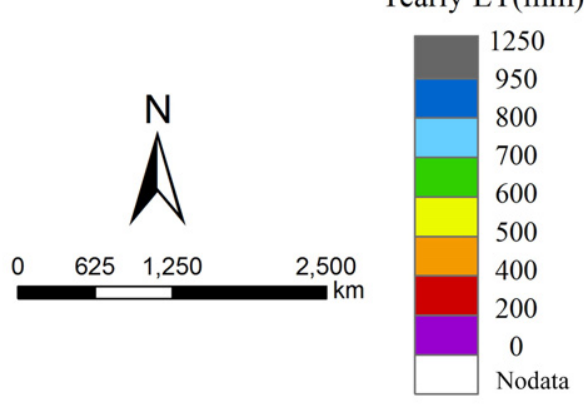

Fig. 2. The ET distribution in China in 1991, 1995 and 1999 .

different physical mechanisms, these methods differentiate in scale representation. Bowen Ratio Energy Balance and Eddy Covariance are constrained to be used in local measurements, because they are expensive to implement, and difficult to maintain in large scales. In order to obtain regional average surface fluxes, a network of stations is needed. LAS can be used to obtain path-averaged surface fluxes over several kilometers, and it is relatively cheap and easy to maintain. However, a disadvantage of the LAS method is that it is based on the semi-empirical Monin-Obukhov similarity theory, while the EC method involves direct measurements. Since path lengths of LAS are comparable to the pixel sizes of satellite images, they enable the validation of ET estimated by remote sensing models with ground observations. In this paper, the study area China is fairly large, and the spatial resolution is relatively coarse, therefore, it is difficult to validate local ET values with the methods mentioned above. Consequently, we adopt two other methods to validate the simulated ET: one is the regional average validation with long-term meteorological observations in typical areas or river; the other is cross-validation with other modeling ET.

\subsubsection{Validation in the experimental area of Changbai Mountains}

The experimental area of Changbai Mountains was selected as the validation area. Part of the observed and simulated regional ET was collected (Feng, 2004), which included four steps: (1) Integrated experiments, mainly referring to field measurement and observations in August and September of 2002 and 2003 respectively, included observations of routine meteorological data, also observations of LAI and net primary productivity (NPP), which are adopted in the estimation. (2) Simulation of NPP and ET with high spatial resolution data using BEPS-China (Boreal Ecosystems Primary Simulator-China) model in the corresponding period. TM-BEPS data are regional ET data simulated by BEPS-China using satellite data such as TM (Landsat Thematic Mapper), ETM (Enhanced Thematic Mapper) and ASTER (Advanced Spaceborne Thermal Emission and Reflection Radiometer), and MODIS-BEPS data are simulated ET basted on 1-km MODIS (Moderate Resolution Imaging Spectroradiometer) data. (3) Validation of the BEPS-China model using field observation data such as observed LAI and NPP. (4) Comparison of TM-BEPS data with MODIS-BEPS data, where TM-BEPS data have better agreement with MODIS-BEPS data according to the ET distribution in the experimental area.

The land cover of the Changbai Mountains were classified into 10 types, which were mixed forest, Erman's birch forest, conifer forest, deciduous broad-leaf forest, tundra, ginseng plant area, cropland area, rural residential area, urban area, and water body. Based on this classification, average ET on different land cover types was identified using TM-BEPS and MODIS-BEPS data respectively in year 2001. In spatial, ET in the Changbai Mountains simulated by TM-BEPS data shows vertical zonality in the year 2001, especially under the different vegetation

Table 1

Comparison of average ET based on TM-BEPS and MODIS-BEPS data over different vegetation cover types in 2001.

\begin{tabular}{lll}
\hline Land cover types & \multicolumn{2}{l}{ Average ET $\left(\mathrm{mm} \cdot \mathrm{a}^{-1}\right)$ in 2001 } \\
\cline { 2 - 3 } & TM-BEPS data & MODIS-BEPS data \\
\hline Needle and broad-leaf mixed forest & 519 & 475 \\
Tundra & 226 & 230 \\
Needle-leaf forest & 345 & 346 \\
Erman's birch forest and broad-leaf forest & 568 & 532 \\
Crops & 446 & 440 \\
Total area & 450.2 & 515.4 \\
\hline
\end{tabular}


cover conditions (Table 1$)$. Where low ET values $\left(<50 \mathrm{~mm} \cdot \mathrm{a}^{-1}\right)$ are mainly distributed in tundra with high elevation, low temperature, sparse vegetation and low evaporation level of soil and vegetation; relatively low ET values (between 50 and $200 \mathrm{~mm} \cdot \mathrm{a}^{-1}$ ) are mainly distributed in tundra and in parts of Erman's birch forest with relatively high elevation; middling ET values (between 200 and $500 \mathrm{~mm} \cdot \mathrm{a}^{-1}$ ) are mainly distributed in regions with coniferous forest and mixed forest; and the highest ET values (up to $802 \mathrm{~mm} \cdot \mathrm{a}^{-1}$ ) are scattered around the Changbai Mountain area. The distribution of ET simulated by MODIS-BEPS data shows a good agreement with the distribution of ET simulated TM-BEPS data. According to the same land cover, ET simulated using the two kinds of data sources, without considering the influence of LUCC, mixed pixel and the temporal difference among remote sensing images, have approximately the same average values.

Considering the compatibility of the different land cover classification, we only compared average ET values in the experimental area in this study. The annual average ET values in the Changbai Mountains simulated by the SEBS-China model is $415 \mathrm{~mm}, 485 \mathrm{~mm}, 412 \mathrm{~mm}$ over year 1991, 1995 and 1999.

The annual average ET simulated by SEBS-China in the Changbai Mountains is $437 \mathrm{~mm}$, which is approaching the annual ET (450.2 mm) simulated by TM-BEPS data for the year 2001, and a little lower than ET ( $515.4 \mathrm{~mm}$ ) simulated by MODIS-BEPS data. Therefore, it can be concluded the simulated results by the SEBS-China model are regionally reasonable.

\subsubsection{Validation in typical river basins}

Five representative river basins were selected to validate regional ET values. Shown in Fig. 3, the river basins are: the Yellow River Basin, the Yangtze River Basin, the Pearl River Basin, the Haihe River Basin and the Liaohe River Basin. Based on the surface water balance law precipitation almost equals the sum of runoff and ET at basin scale under the multiannual average level (Wang and Dickinson, 2012). Thus, based on runoff and precipitation data from hydrological stations in these basins, the annual average ET values between 1991 and 1999 can be calculated. Table 2 shows the comparison between ET values calculated and simulated by the SEBS-China model.

The measured ET values shown in Table 2 are calculated from precipitation and runoff in the five representative basins and can indirectly represent the multi-annual average level of ET from the last ten years. As it can be seen, the simulated ET values in the Yellow and Haihe River Basins are slightly higher than the measured ones. The measured average ET values for all the investigated river basins changed from an increasing trend from 1991 to 1995 to a decreasing trend from 1995 to 1999 , which is consistent with long term changing trend of the regional climate of China. As shown in Fig. 4, the Yellow River has the maximum error between the simulated and measured annual average ET values in the recent ten years, where the maximum error reaches $221 \mathrm{~mm}$ and the relative error is about 58\%. The errors of the other four basins are relatively small with $82 \mathrm{~mm}$ and $14 \%$ for the Yangtze River; $77 \mathrm{~mm}$ and 11\% for the Pearl River; $145 \mathrm{~mm}$ and 36\% for the Haihe River and $6 \mathrm{~mm}$ and 1.5\% for the Liaohe River.

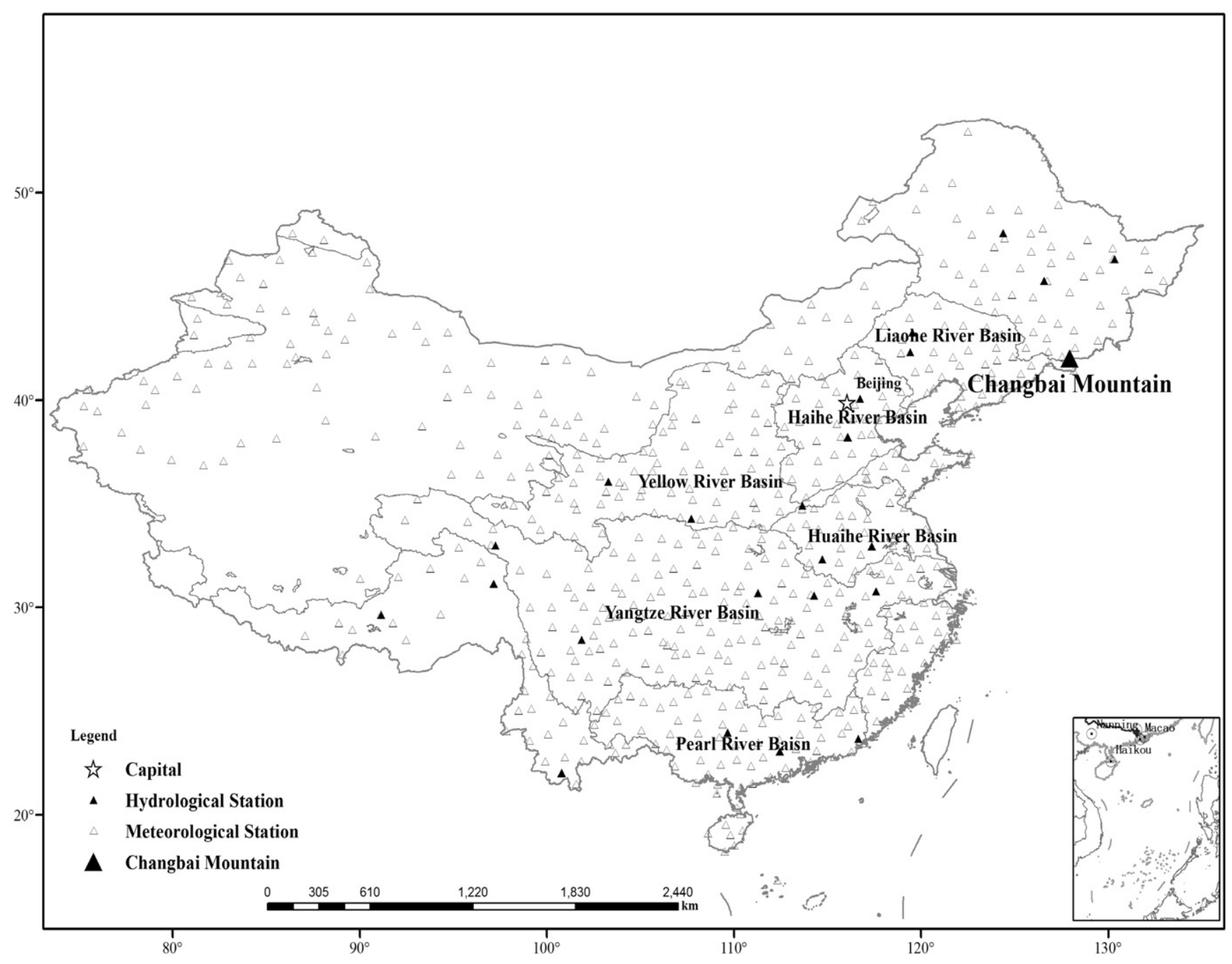

Fig. 3. Spatial distribution of representative river basins in China. 
Table 2

Comparison of annual average ET values in representative river basins.

\begin{tabular}{|c|c|c|c|c|c|c|c|}
\hline \multirow[t]{2}{*}{ River basin } & \multirow[t]{2}{*}{ Area $\left(10^{4} \mathrm{~m}^{2}\right)$} & \multicolumn{3}{|c|}{ Observed water balance parameters } & \multicolumn{3}{|c|}{ Simulated $\mathrm{ET}_{\text {SEBS }}(\mathrm{mm})$} \\
\hline & & Precipitation (mm) & Runoff (mm) & $\mathrm{ET}(\mathrm{mm})$ & 1991 & 1995 & 1999 \\
\hline Yangtze River & 180.7 & 1100 & 525 & 575 & 642 & 727 & 601 \\
\hline Yellow River & 75.2 & 456 & 77 & 379 & 619 & 633 & 547 \\
\hline Pearl River & 44.4 & 1469 & 752 & 717 & 640 & 695 & 584 \\
\hline Haihe River & 26.4 & 499 & 103 & 396 & 570 & 577 & 476 \\
\hline Liaohe River & 22.9 & 473 & 65 & 408 & 397 & 461 & 384 \\
\hline
\end{tabular}

\subsubsection{Cross-validation with CoLM modeling}

CoLM (Common Land Model) is the latest generation land surface process model, which was developed from a great deal of work, particularly on the numerical simulation of the climate, vegetation, ecology and hydrology processes (Dai et al., 2003). It has integrated most of the current land surface models' merits, considered the interaction among atmosphere, land, ocean, and ocean-sea ice, and also added some new land surface processes, such as surface runoff, biological, geographical and chemical processes, vegetation dynamics and carbon cycle. Based on the grid size of CoLM, the average relative error of the three years $(1991,1995$, and 1999$)$ is respectively: $8.6 \%, 8.5 \%$, and $7.9 \%$; the root mean square error is respectively: $126.6 \mathrm{~mm} \cdot \mathrm{a}^{-1}$, $131.2 \mathrm{~mm} \cdot \mathrm{a}^{-1}$, and $123.8 \mathrm{~mm} \cdot \mathrm{a}^{-1}$; the correlation coefficient respectively: $0.878,0.905$, and 0.931 with statistically significant at the 0.01 level. Fig. 5 shows SEBS-China's simulated average monthly ET distribution in China in January, April, July, and October 1991, also shows the average monthly ET distribution in the corresponding periods simulated by CoLM.

The simulated results show that ET values of the northwest part of China are larger than actual ET values in the same area, especially in the Qinghai-Tibet Plateau, where the monthly actual ET value is less than $10 \mathrm{~mm}$ (and even less than $2 \mathrm{~mm}$ in some months) and relatively high (even more than $15 \mathrm{~mm}$ ) between May and August. According to Fig. $5 a-d$, it is calculated that the SEBS-China-based average ET values of China in January, April, July and October are about $20 \mathrm{~mm}, 40 \mathrm{~mm}$, $80 \mathrm{~mm}$ and $50 \mathrm{~mm}$ respectively. The fluctuating trend is reasonable, but the simulated ET values are slightly higher than the actual ones.

As shown in Fig. 5e-h, the fluctuating trend of the average monthly ET values simulated by CoLM is close to the SEBS-China-based ones. Most of the monthly ET values are below $40 \mathrm{~mm}$, while in the south of the Qinghai-Tibet Plateau, on Yunnan-Guizhou Plateau and in southeastern coastal areas, the ET values are very high. In the north of Heilongjiang, the north of Xinjiang and the north of the Qinghai-Tibet Plateau, the ET values are very low in January. In January and April the ET values simulated by CoLM are very close to those by SEBS-China. In January, April, July and October the average monthly ET values in the Pearl River Basin a little higher than the other basins In October, the high values (above $75 \mathrm{~mm}$ ) simulated by CoLM are concentrated in

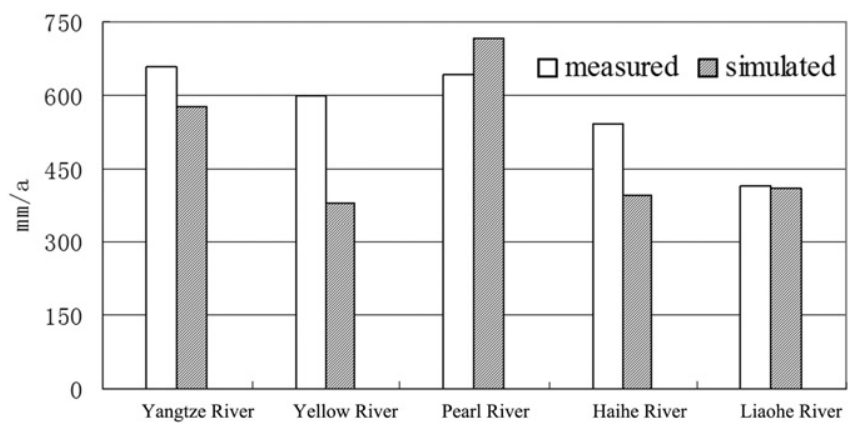

Fig. 4. Comparison of the measured and simulated annual average ET of representative river basins. the south of China and on the Yunnan-Guizhou Plateau. Most of ET values in the northwest are below $10 \mathrm{~mm}$, which is reasonable under China's climate condition.

In general, the ET values from the two models show the same fluctuating trend following the seasonal changes. Compared with CoLM, SEBS-China can provide higher accuracy of spatial resolution ET results. The fluctuating trend shown in Fig. 5e-h is similar to that in Fig. 5a-d over China.

\section{Discussions and conclusions}

The original SEBS model was revised and developed in this paper according to the actual regional characteristics of China to be appropriate for estimating China's regional ET. The simulated ET regional distribution generally has a good agreement with the similar model test in other researches (Chen et al., 2014; Liu et al., 2012; Thomas, 2008; Xiao et al., 2013). The simulated ET results show that the Yangtze and Pearl rivers locate in high ET regions, the Liaohe River mainly locates in the medium ET regions, the Yellow and Haihe rivers cross the medium and low ET regions, but the simulated ET in some places is significantly higher such as south of the Qinghai-Tibet Plateau. The reason is that the mechanism of SEBS-China is mainly based on the energy balance, while the CoLM's mechanism has a combination of energy balance and water balance, which is more reasonable for the place lack of meteorological and hydrological data. The simulated ET regional distribution generally has a good agreement with actual regional ET, and with more accurate spatial resolution than the CoLM-based data. The simulation results proved that the improved SEBS, or SEBS-China, is more appropriate for estimating China's regional ET.

Three methods were applied in the regional validation of ET. Firstly, the annual average ET values from the SEBS-China model for the Changbai Mountains are found similar to the ET values simulated by TM-BEPS data and MODIS-BEPS data, making the SEBS-China model regionally reasonable. Secondly, the five representative river basins in China were also selected to verify regional ET values. Through verifications, it was found that the simulated average annual ET value of the Yellow River was much larger than the observation result of the other four basins, whose simulation errors are relatively small. Thirdly, a model cross-validation was done between SEBS-China and CoLM. The results indicated that the two models can simulate regional ET effectively except that CoLM overestimates ET values in some areas of the YanShan Mountains and the southern Daxing'anling Mountains in July, while SEBS-China overestimated the ET values for the northwest, in places like the Qinghai-Tibet Plateau, where the monthly actual ET value is less than $10 \mathrm{~mm}$ (and even less than $2 \mathrm{~mm}$ in some months) and relatively high (even more than $15 \mathrm{~mm}$ ) between May and August. Such a phenomenon is related to the local sum of precipitation, for there is a lot of rainfall between May and August. The maximum air temperature and ET value also appear within this period. In September the precipitation level and air temperature begin to decrease, a trend that stays until the following April, while ET remains minimal. In short, uncertainties exist in both models, making the cross-validation with different models to improve the accuracy of simulated ET feasible. 

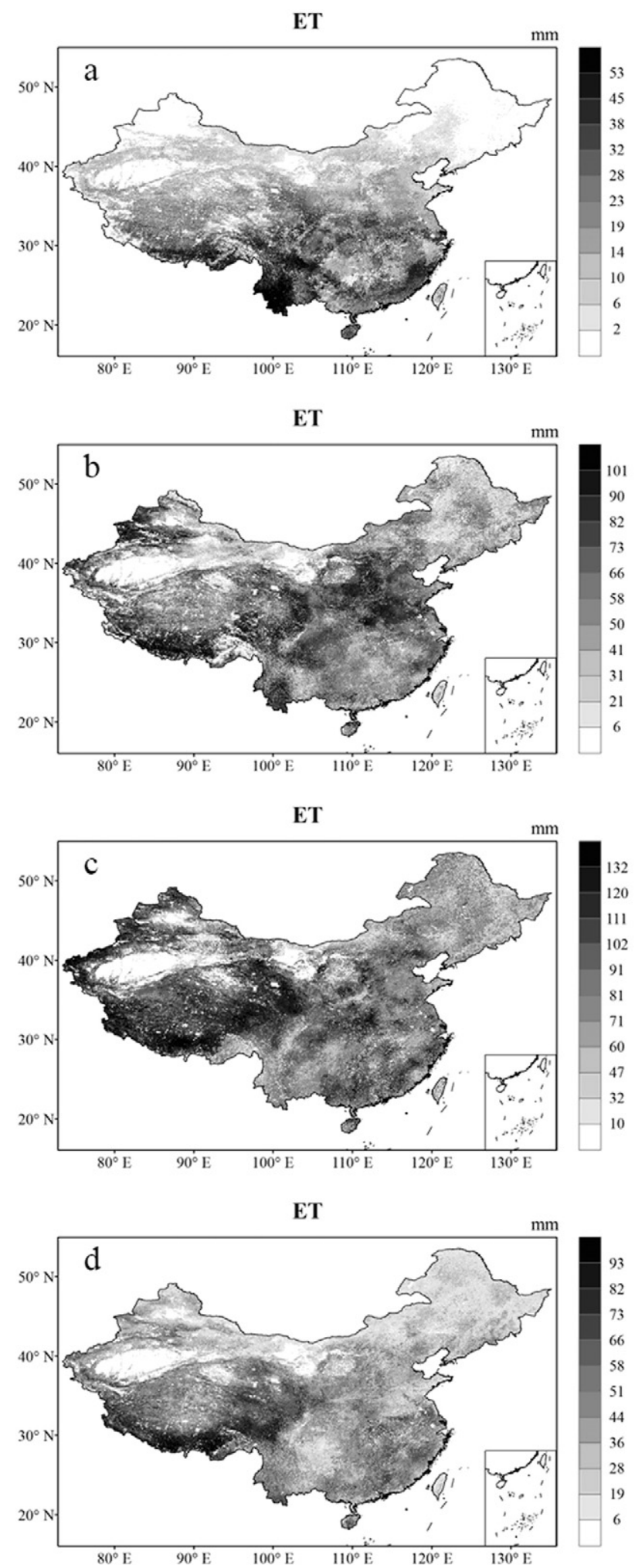
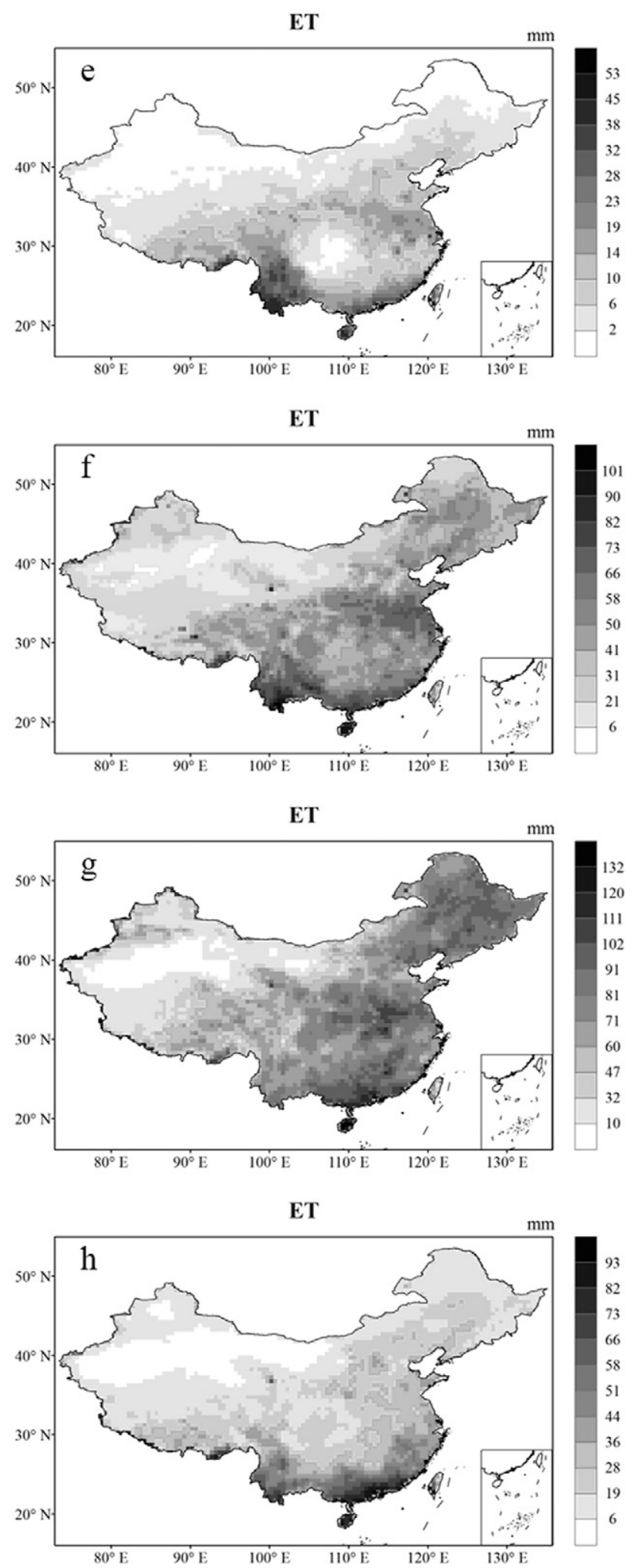

Fig. 5. ET simulated by SEBS in Jan. 1991 (a), Apr. 1991 (b), Jul. 1991 (c), and Oct. 1991 (d), and simulated by CoLM in Jan. 1991 (e), Apr. 1991 (f), Jul. 1991 (g), and Oct. 1991 (h).

There were still urgent problems to be solved in data acquisition, modeling parameterization, model processes and mechanism improvements, such as:

Some of SEBS-China's sub-models still need improvements, among which are the LAI model, the fractional vegetation coverage model and the total net radiation model. The time scaling-up model to ET using fractional evaporation also needs further improvements. In addition, the error comparisons in Fig. 4 show that the simulated results in moist and semi-moist areas are better than those in arid and semiarid areas. The explanation lies in the model limitation of the onelayer mechanism, which is not suitable for ET modeling for sparse vegetation areas. Therefore, there are larger simulation errors for the Yellow River and the Haihe River, and better simulation for the Yangtze River, the Pearl River, and the Liaohe River that are all located in moist 
and semi-moist areas. There is a large area of arid and semi-arid region in China, where the SEBS model with a one-layer mechanism may be not more suitable than with the two-layer mechanism which considers the evaporation and transpiration respectively. So the simulation for arid and semi-arid areas with SEBS-China is still deficient and it is necessary to develop the SEBS-China to consider a two-layer mechanism.

A large-scale experimental field for land surface flux validation should be established so as to validate fluxes not only at points, but also within regions. However, a lot of the relevant measured data in China is not currently based on uniform space-time standards. Consequently, much more work should be concentrated on multidimensional data assimilation.

Regional ET simulation by remote sensing models is efficient, but has some disadvantages. For a large space, where underlying surface is complex, a multitude of factors including all the physical, biological, and hydrological processes and their interactions should be considered. The land surface process model has its inherent advantages over remote sensing models, but the land surface process model is insufficient for regional ET simulation. The combination of the two methods will help to improve the accuracy of ET estimation and reduce the simulation uncertainties.

\section{Acknowledgments}

This work was partially supported by the National Basic Research Program of China ("973" Program) (2015CB452701), the Natural Science Foundation of China (41271003, 41401042), China Postdoctoral Science Foundation (2014 M550823) and Anhui Provincial Natural Science Foundation (1508085QD69). The authors are grateful to the reviewers for the help and thought-provoking comments.

\section{References}

Acharya, A., Piechota, C.T., Stephen, H., Tootle, G., 2011. Modeled streamflow response under cloud seeding in the North Platte River watershed. J. Hydrol. 409, 305-314.

Bastiaanssen, W.G.M., 1995. Regionalization of Surface Flux Densities and Moisture Indicators in Composite Terrain. (Dissertation). Wageningen Agriculture University.

Bastiaanssen, W.G.M., Allen, R.G., Droogers, P., D'Urso, G., Steduto, P., 2007. Twenty-five years modeling irrigated and drained soils, state of the art. Agric. Water Manag. 92, 111-125.

Brutsaert, W., 1999. Aspects of bulk atmospheric boundary layer similarity under freeconvective conditions. Rev. Geophys. 37, 439-451.

Chen, Y., Xia, J.Z., Liang, S.L., et al., 2014. Comparison of satellite-based evapotranspiration models over terrestrial ecosystems in China. Remote Sens. Environ. 140, 279-293.

Ching, J.K.S., 2013. A perspective on urban canopy layer modeling for weather, climate and air quality applications. Urban Clim. 3, 13-39.

Choudhury, B.J., Fung I.Y., 1989. Satellite observed global vegetation dynamics and its relations with biosphere-atmosphere carbon exchange. Adv. Space Res. 9, 229-237.

Choudhury, B.J., Monteith, J.L., 1988. A four layer model for the heat budget of homogeneous land surfaces. Q. J. R. Meteorol. Soc. 114, 373-398.

Courault, D., Seguin, B., Olioso, A., 2003. Review to estimate evapotranspiration from remote sensing data: some examples from the simplified relationship to the use of mesoscale atmospheric models. ICID Workshop on Remote Sensing of ET for Large Regionspp. 1-18.

Dai, Y.J., Zeng, X.B., Dickinson, R.E., et al., 2003. The Common Land Model (CLM). Bull. Am. Meteorol. Soc. 84, 1013-1023.

Ershadi, A., McCabe, M.F., Evans, J.P., Chaney, N.W., Wood, E.F., 2014. Multi-site evaluation of terrestrial evaporation models using FLUXNET data. Agric. For. Meteorol. 187 46-61.

Feng, X.F., 2004. Simulating Net Primary Productivity and Evapotranspiration of Terrestrial Ecosystems in China Using a Process Model Driven by Remote Sensing. (Dissertation). Institute of Geographical Sciences and Natural Resources Research, Chinese Academy of Sciences, China.

Gokmen, M., Vekerdy, Z., Verhoef, A., Verhoef, W., Batelaan, O., van der Tola, C., 2012. Integration of soil moisture in SEBS for improving evapotranspiration estimation under water stress conditions. Remote Sens. Environ. 121, 261-274.
Gottschalck, J., Meng, J., Rodell, M., et al., 2005. Analysis of multiple precipitation products and preliminary assessment of their impact on global land data assimilation system land surface states. J. Hydrometeorol. 6, 573-598.

Huang, X., Lyons, T.J., Smith, R., Hacker, J.M., Schwerdtfeger, P., 1993. Estimation of surface energy balance from radiant surface temperature and NOAA AVHRR sensor reflectances over agricultural and native vegetation. J. Appl. Meteorol. 32, 1441-1449.

Jung, M., Reichstein, M., Ciais, P., Seneviratne, S., Sheffield, J., Goulden, M., et al., 2010. Recent decline in the global land evapotranspiration trend due to limited moisture supply. Nature 467, 951-954.

Kustas, W.P., Norman, J.M., 1996. Use of remote sensing for evapotranspiration monitoring over land surfaces. Hydrol. Sci. J. 41, 495-516.

Kustas, W.P., Choudhury, B.J., Moran, M.S., 1989. Determination of sensible heat flux sparse canopy using thermal infrared data. Agric. For. Meteorol. 44, 197-216.

Kustas, W.P., Norman, J., Anderson, M., French, A., 2003. Estimating subpixel surface temperatures and energy fluxes from the vegetation index-radiometric temperature relationship. Remote Sens. Environ. 85, 429-440.

Lhomme, J.P., Monteny, B., Amadou, M., 1994a. Estimating sensible heat flux from radiometric temperature over sparse millet. Agric. For. Meteorol. 68, 77-91.

Lhomme, J.P., Monteny, B., Chehbouni, A., 1994b. Determination of sensible heat flux over Sahelian fallow savannah using infrared thermometry. Agric. For. Meteorol. 68, 93-105.

Li, Z.L., Tang, R.L., Wan, Z.M., et al., 2009. A review of current methodologies for regional evapotranspiration estimation from remotely sensed data. Sensors 9, 3801-3853.

Liu, J.Y., Liu, M.L., Zhuang, D.F., et al., 2003. Study on spatial pattern of land-use change in China during 1995-2000. Sci. China (Ser. D) 46, 1373-1384.

Liu, M.L., Tian, H.Q., Lu, C.Q., et al., 2012. Effects of multiple environment stresses on evapotranspiration and runoff over eastern China. J. Hydrol. 426-427, 39-54.

Liu, J.Y., Kuang, W.H., Zhang, Z.X., Xu, X.L., et al., 2014. Spatiotemporal characteristics, patterns and causes of land-use changes in China since the late 1980s. J. Geogr. Sci. 2014 (24), 195-210.

Ma, W.Q., Ma, Y.M., Hirohiko, I., 2014. Evaluation of the SEBS for upscaling the evapotranspiration based on in-situ observations over the Tibetan Plateau. Atmos. Res. 138, 91-97.

McMahon, T.A., Peel, M.C., Lowe, L., Srikanthan, R., McVicar, T.R., 2013. Estimating actual, potential, reference crop and pan evaporation using standard meteorological data, a pragmatic synthesis. Hydrol. Earth Syst. Sci. 17, 1331-1363.

Menenti, M., Choudnury, B.J., 1993. Parametrization of land surface evapotranspiration using a location-dependent potential evapotranspiration and surface temperature range. In: Bolle, H.J., et al. (Eds.), Exchange Processes at the Land Surface for a Range of Space And time Scales. IAHS Publ 212, pp. 561-568.

Moran, M.S., Clarke, T.R., Inoue, Y., 1994. Estimating crop water deficit using the relation between surface-air temperature and spectral vegetation index. Remote Sens. Environ. 49, 246-263.

Moran, M.S., Rahman, A.F., Washburne, J.C., et al., 1996. Combining the Penman-Monteith equation with measurements of surface temperature and reflectance to estimate evaporation rates of semiarid grassland. Agric. For. Meteorol. 80, 87-109.

Norman, J.M., Kustas, W.P., Humes, K.S., 1995. A two-source approach for estimating soil and vegetation energy fluxes from observation of directional radiometric surface temperature. Agric. For. Meteorol. 77, 263-293.

Su, Z., 2000. Remote sensing of land use and vegetation for mesoscale hydrological studies. Int. J. Remote Sens. 21, 213-233.

$\mathrm{Su}, \mathrm{Z}$., 2002. The Surface Energy Balance System (SEBS) for estimation of turbulent heat fluxes. Hydrol. Earth Syst. Sci. 6, 85-89.

Su, Z., Jacobs, C., 2001. Advanced Earth Observation - Land Surface Climate, Publications of the National Remote Sensing Board. BCRS, USP-2, p. 184.

Su, Z., Menenti, M., 1999. Mesoscale Climate Hydrology, The Contribution of the New Observing Systems, Report USP-2, 99-05, Publications of the National Remote Sensing Board. BCRS, p. 141

Su, Z., Pelgrum, H., Menenti, M., 1999. Aggregation effects of surface heterogeneity in land surface processes. Hydrol. Earth Syst. Sci. 3, 549-563.

Thomas, A., 2008. Development and properties of 0.25-degree gridded evapotranspiration data fields of China for hydrological studies. J. Hydrol. 358, 145-158.

Wang, K.C., Dickinson, R.E., 2012. A review on global terrestrial evapotranspiration: observation, modeling, climatology, and climatic variability. Rev. Geophys. 50, RG2005. http://dx.doi.org/10.1029/2011RG000373.

Wood, E.F., Su, H., McCabe, M., Su, Z., 2003. Estimating evaporation from satellite remote sensing. Paper Presented at the IGARSS 7, pp. 21-25.

Xiao, J.F., Sun, G., Chen, J.Q., et al., 2013. Carbon fluxes, evapotranspiration, and water use efficiency of terrestrial ecosystems in China. Agric. For. Meteorol. 182-183, 76-90.

Zhan, C.S., Xia, J., Chen, Z., Li, Z.L., Xu, Z.X., 2007. Remote sensing estimation of land surface evapotranspiration of typical river basins in China. Proceedings of the IAHS Scientific Assembly, 220-227. IAHS Publication, Perugia. 